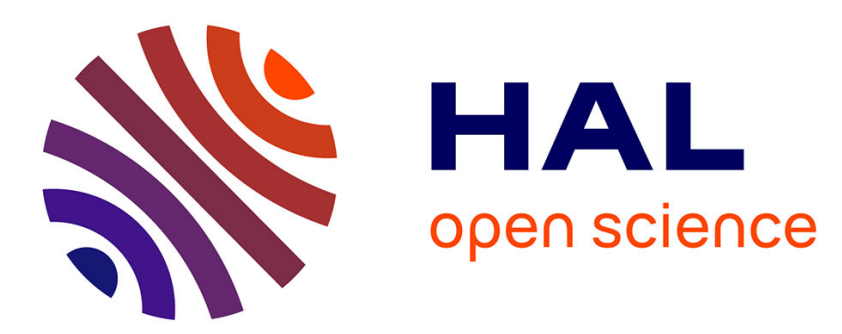

\title{
How methane hydrate recovers at very high pressure the hexagonal ice structure
}

\author{
S. Schaack, Ph. Depondt, M. Moog, F. Pietrucci, Fabio Finocchi
}

\section{To cite this version:}

S. Schaack, Ph. Depondt, M. Moog, F. Pietrucci, Fabio Finocchi. How methane hydrate recovers at very high pressure the hexagonal ice structure. Journal of Chemical Physics, 2020, 152 (2), pp.024504. 10.1063/1.5129617 . hal-03029341

\section{HAL Id: hal-03029341 https://hal.science/hal-03029341}

Submitted on 28 Nov 2020

HAL is a multi-disciplinary open access archive for the deposit and dissemination of scientific research documents, whether they are published or not. The documents may come from teaching and research institutions in France or abroad, or from public or private research centers.
L'archive ouverte pluridisciplinaire $\mathbf{H A L}$, est destinée au dépôt et à la diffusion de documents scientifiques de niveau recherche, publiés ou non, émanant des établissements d'enseignement et de recherche français ou étrangers, des laboratoires publics ou privés. 


\section{How methane hydrate recovers at very high pressure the hexagonal ice structure}

S. Schaack, ${ }^{1} \mathrm{Ph}$. Depondt, ${ }^{1}$ M. Moog, ${ }^{2}$ F. Pietrucci, ${ }^{2}$ and F. Finocchi ${ }^{1, \text { a) }}$

1) Sorbonne Université, Institut des Nanosciences de Paris (INSP), CNRS UMR 7588, Paris, France

2) Sorbonne Université, Institut de minéralogie, de physique des matériaux et de cosmochimie (IMPMC), CNRS UMR 7590, Paris, France

(Dated: 8 December 2019)

Methane hydrate was recently shown, both experimentally and through simulations, to be stable up to the remarkably high pressure of $150 \mathrm{GPa}$. A new methane hydrate high-pressure (MH-IV) phase, reminiscent of ice at ambient pressure, was described for pressures above approximately $40 \mathrm{GPa}$. We disentangle here the main contributions to the relative stability of the lower pressure, denoted MH-III and the high-pressure MH-IV structures. Through several simulation techniques, including metadynamics and Path Integral Molecular Dynamics for nuclear quantum effects, we analyze the phase transition mechanism, which implies hydrogen bond breaking and reforming, as well as methane reordering. The transition pathway is far from trivial and the quantum delocalization of the hydrogen nuclei plays a significant role.

Keywords: Phase transitions, Methane Hydrate, High pressures, Nuclear Quantum Effects, Ab initio, Metadynamics

\section{INTRODUCTION}

Gas clathrate hydrates consist of non-polar molecules, such as methane, carbon dioxide, hydrogen, nitrogen, that are enclosed within a network of hydrogen-bonded water molecules ${ }^{1}$. They can form spontaneously in nature, although the kinetics of their formation is largely unknown and only recently has been addressed ${ }^{2,3}$. Hydrates of non-polar molecules and rare-gas atoms are often described theoretically as water molecules forming a tetrahedral network in a straddling mode around the enclosed molecules ${ }^{4}$. As a function of size, form and number of the enclosed molecules, there are several possible arrangements for the water network, which often consists of pentagonal and hexagonal rings forming a variety of polyhedra ${ }^{5}$. As those motifs are recurrent in many different clathrates, current research tries to understand how the interplay between the attractive water-water interaction within the framework and the repulsive watermolecule interaction could favor a particular structure upon the others. The phase diagram of clathrate hydrates, although partly unexplored, is fairly well assessed at pressures below $1 \mathrm{GPa}^{1,6}$. The decomposition of gas clathrates into their elemental constituents was expected at high pressures. Notwithstanding this widespread belief, it was shown that many stable hydrates can form at pressures exceeding $1 \mathrm{GPa}^{7}$. However, there are several open issues about the stability of hydrates beyond the previous limit, mainly concerning the existence of new structures and their survival up to extreme pressures ${ }^{1}$. In this regime, the kinetics of formation and the transition mechanisms between high-pressure hydrate phases are largely unexplored.

In the present paper, we address the transformation between two phases of methane hydrate, phase III and

a)Electronic mail: fabio.finocchi@insp.jussieu.fr the recently discovered high-pressure (IV) phase $^{8}$, which occurs around $40 \mathrm{GPa}$. The orthorhombic phase III of methane hydrate (MH-III) was synthesized and shown to survive up to about $12 \mathrm{GPa}^{9}$ and even further ${ }^{10}$. The new stable phase of methane hydrate at high pressure (MHIV) was recently discovered by joint Raman spectroscopy and first-principle simulations including nuclear quantum effects $^{8}$ and is consistent with up to then unresolved Xray scattering results ${ }^{11}$. It is characterized by a water network which is isomorph with ordinary hexagonal ice, where the $\mathrm{CH}_{4}$ molecules are aligned along the $c$ axis. Why does the ice network recover its tetrahedral-based network with 6 -member rings only? To the best of our knowledge, hydrates have a different arrangement from ordinary ice. Is this specific to methane hydrate? Is methane a structural catalyst for hydrates at very high pressure?

In order to address those issues, we compare the structures of MH-III and MH-IV and analyze their relative stability as a function of the applied pressure $P$ by splitting the enthalpy difference in terms of four contributions: the water-water interaction, the methanemethane-interaction, the interaction between the host methane and the ice frame, and the $P \Delta V$ term. We break up both ice networks as a sum of contributions from the different rings forming the two phases, and their stability with pressure. Then, we analyze the transformation path between MH-III to $\mathrm{MH}-\mathrm{IV}$, which reveals several interesting facts: (i) deuteron tunneling through hydrogen bonds triggers the rearrangement of the ice network; (ii) the specific features of the barrier crucially depend on the theory level, specifically on the inclusion of temperature and nuclear quantum effects. The role of the methane molecules, which are not simple spectators in this transformation, is critically analyzed. 


\section{METHODS}

The total-energy and force calculations were done by means of the Quantum Espresso package ${ }^{12}$, in the framework of the Density Functional Theory (DFT). We employed the Generalized Gradient approximation $(\mathrm{GGA})^{13}$ to the DFT. Van der Waals interactions were added by following the semi-empirical scheme by Grimme ${ }^{14}$. We used ultra-soft pseudopotentials to represent the nuclei and 1s core electrons of $\mathrm{O}$ and $\mathrm{C}$ ions, as well as for the deuterons. The kinetic energy cutoffs on the plane waves for the expansions of the wave functions and the electronic charge and potential are $50 \mathrm{Ry}$ and $400 \mathrm{Ry}$, respectively. The simulated samples consist of 16 methane molecules and 32 water molecules in $2 \times 1 \times 2$ orthorhombic supercell, for both MH-III and MH-IV phases. The corresponding Brillouin zones are sampled with a $3 \times 1 \times 2$ grid centred at the $\Gamma$ point. The ensemble of these approximations were extensively checked.

The methane molecules are hydrogenated $\left(\mathrm{CH}_{4}\right)$ while the ice cages consist of deuterated $\left(\mathrm{D}_{2} \mathrm{O}\right)$ water molecules. As far as MH-III is concerned, the initial structure was taken from ref. 7 and then optimized, by letting both lattice parameters and atomic positions relax. We also run a series of $10 \mathrm{ps}$ long dynamical simulations in the $N V T$ ensemble. After each dynamical run, the mean internal pressure was computed and the lattice parameters for the next simulation were varied by a trial-and-error procedure. Three or four simulations were generally enough to ensure that the mean total internal pressure of the last run matched the external one within $\pm 1 \mathrm{GPa}$. In this way, we account for finite temperature effects on the equilibrium volume and lattice parameters as pressure increases. We refined the high-pressure methane hydrate configurations through a series of structural optimizations, starting from several educated guesses. Along with structural optimization, we adopted a similar strategy to that described above for MH-III to obtain the target internal pressure, including thermal effects.

On top of DFT energy and force calculations, we used several methods to explore the MH-IV to MH-III transition. First, the nudged elastic band (NEB) method, in its climbing-image version, as implemented in the Quantum Espresso package yields a 0K transition mechanism without NQE. Second, metadynamics, in which we chose the collective variable by relying on the topological distance between the MH-III and the MH-IV configurations ${ }^{15}$. Third, simulations that are based on the path-integral formalism in conjunction with a generalized Langevin equation (PIGLET implementation) ${ }^{16}$ allowed us to estimate the importance of nuclear quantum effects along the transition path. All the dynamical simulations have been carried out at $300 \mathrm{~K}$. Further details on the sequence of configurations along the path in the three methods are given in section IV.

\section{PHASES III AND IV OF METHANE HYDRATE}

In both phases III and IV of methane hydrates, the water-to-methane theoretical ratio is 2 : the unit formula
TABLE I. Lattice parameters $a, b, c$ in $\AA$ and corresponding volume $V$ of the unit cell (for MH-III and MH-IV at $P=40$ GPa, as obtained through structural optimization at $T=0 \mathrm{~K}$. Zero-point effects are not included.

\begin{tabular}{llllr}
\hline \hline structure & $a(\AA)$ & $b(\AA)$ & $c(\AA)$ & $V\left(\AA^{3}\right)$ \\
\hline MH-IV & 4.063 & 6.981 & 6.063 & 171.97 \\
MH-III & 4.006 & 6.911 & 6.249 & 172.99 \\
\hline \hline
\end{tabular}

is thus $\mathrm{CH}_{4}:\left(\mathrm{D}_{2} \mathrm{O}\right)_{2}$. Both structures are orthorhombic; their lattice parameters and unit-cell volume are reported in Table I. Throughout the explored pressure range (5 $\mathrm{GPa} \leq P \leq 150 \mathrm{GPa})$, the MH-IV phase is slightly denser than MH-III.

In MH-III the deuterated water network consists of 4-fold, 6-fold and 8-fold rings. The latter form cavities where two non-equivalent methane molecules take place; the corresponding $\mathrm{C}$ atoms are displaced along the $b$ axis and the methane molecules have four distinct orientations (Fig. 1, upper right panel). Whereas at relatively low pressures $(P \leq 5 \mathrm{GPa})$, the $\mathrm{CH}_{4}$ molecules are rather free to change their orientation, under increasing pressure the methane-water repulsive interaction grows and the $\mathrm{CH}_{4}$ molecules lock-in and progressively distort off of the tetrahedral configuration. Above $P \sim 20 \mathrm{GPa} \mathrm{MH}-$ III forms a crystal compound where water and methane are strongly entangled; moreover, deuterons can tunnel along the hydrogen bond in the water network. Tunneling becomes massive around $30 \mathrm{GPa}$, eventually leading to the deuteron centering between the $\mathrm{O}$ atoms in the ice rings.

In contrast, MH-IV has only six-fold ice rings, equally shared between "armchair" and "boat" types (Fig. 1, lower left and lower right panels, respectively). Therefore, the ice frame shows hexagonal channels along both $\hat{a}$ and $\hat{c}$ directions, where the methane molecules are arranged. They align with a $\mathrm{C}-\mathrm{H}$ bond either parallel or anti-parallel to $\hat{c}$, which is a ternary axis with respect to the sequence of methane molecules (Fig. 1, lower left panel). The other $\mathrm{C}-\mathrm{H}$ bonds point towards the voids in the hexagonal ice rings. When pressure increases, the $c$ axis shrinks and so does the C-C distance; as a result, the $\mathrm{C}-\mathrm{H}$ bond parallel to $c$ becomes progressively shorter than the others, the bond length differences increasing with the applied pressure.

For both MH-III and MH-IV, we compute the distinct contributions to their enthalpy at $T=0 \mathrm{~K}$ as follows. First, we optimize the structures and the lattice parameters at various pressures; from the fully optimized methane hydrates structures (whose computed internal energy is $E_{(\mathrm{MH})}$, we take away: (i) the $\mathrm{CH}_{4}$ molecules and compute the ice-ice interaction energy $E_{\left(\mathrm{H}_{2} \mathrm{O}-\mathrm{H}_{2} \mathrm{O}\right)}$; (ii) the ice frame and compute the methane-methane interaction energy $E_{\left(\mathrm{CH}_{4}-\mathrm{CH}_{4}\right)}$. Both calculations are carried out by clamping the ion positions and lattice parameters to their values in the fully optimized structures. The ice-methane interaction energy is computed through the difference: $E_{\left(\mathrm{H}_{2} \mathrm{O}-\mathrm{CH}_{4}\right)}=E_{(\mathrm{MH})}-E_{\left(\mathrm{H}_{2} \mathrm{O}-\mathrm{H}_{2} \mathrm{O}\right)}-$ $E_{\left(\mathrm{CH}_{4}-\mathrm{CH}_{4}\right)}$. The term $P V$ is added to the internal energies to recover the corresponding $T=0 \mathrm{~K}$ enthalpy 


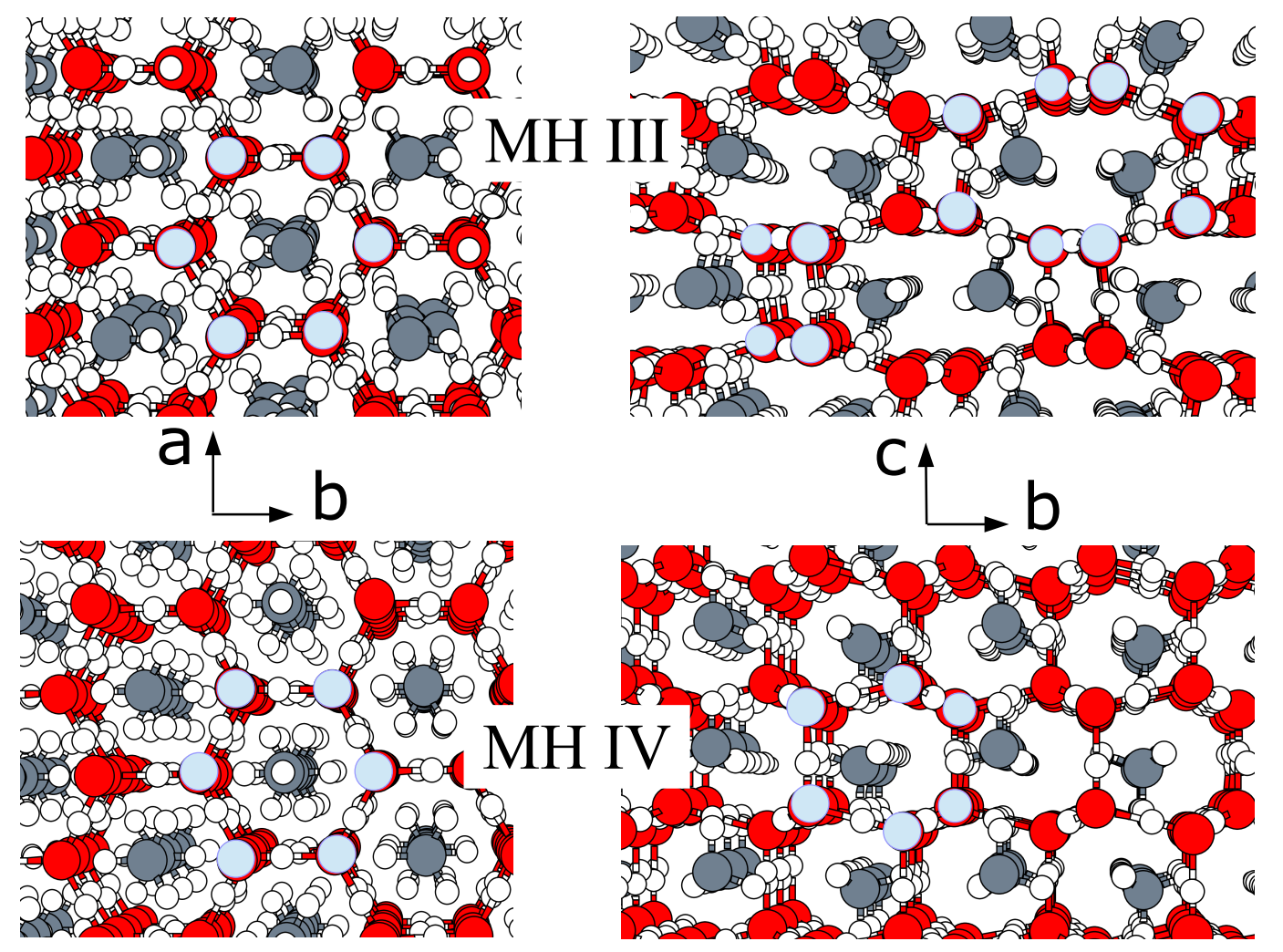

FIG. 1. Structure of methane hydrate at $P=40 \mathrm{GPa}$ : phase III (upper panels) and high-pressure (IV) phase (lower panels). $\mathrm{O}$ are red, $\mathrm{H}$ white, $\mathrm{C}$ dark gray. Left panels: $\mathrm{MH}$ structures in the $(a b)$ plane; armchair 6-fold $\mathrm{O}$ rings are highlighted in cyan. Right panels: MH structures in the $(b c)$ plane; 4-fold and 8-fold rings in MH-III as well as boat 6-fold O rings in MH-IV are highlighted in cyane. Note the distinct arrangement of the methane molecules in the $(a b)$ plane (left), easily seen when comparing the $\mathrm{CH}_{4}$ positions between the two panels on the left. Methane ordering along $a$ is less affected by the MH-III to MH-IV transition, as it can be understood by comparing the two panels on the right.

$H_{(\mathrm{MH})}=E_{(\mathrm{MH})}+P V_{(\mathrm{MH})}$.

In Fig.2, we report energy differences between the MH-III and MH-IV structures. One observes that all terms have the same order of magnitude and that the differences are less than a few tenths of electron-Volts per unit formula $\mathrm{CH}_{4}:\left(\mathrm{D}_{2} \mathrm{O}\right)_{2}$ : the result will thus be the effect of a rather subtle balance. Indeed, the term related to the ice cage $\Delta E_{\left(\mathrm{H}_{2} \mathrm{O}-\mathrm{H}_{2} \mathrm{O}\right)}$ is positive and therefore favors the MH-IV structure at all pressures; the methane-methane term $\Delta E_{\left(\mathrm{CH}_{4}-\mathrm{CH}_{4}\right)}$ is also positive with the same effect but weaker. Conversely, the cage-molecule term $\Delta E_{\left(\mathrm{H}_{2} \mathrm{O}-\mathrm{CH}_{4}\right)}$ is negative thus favoring the MH-III structure. The sum of these energies $\Delta E_{t o t}$ is negative in the pressure range of this work, but, if the pressure energy term $P \Delta V$ is included to obtain the enthalpy difference $\Delta H$, the MH-IV structure is favored from approximately $30 \mathrm{GPa}$ upwards. The slope of $\Delta H$ as a function of pressure is gentle, meaning the entropy related to methane orientational disorder may shift the transition pressure significantly upwards at finite temperature. Indeed, we note that the locking-in of methane molecules should significantly contribute in reducing the stabilizing configurational entropy as pressure is increased in the MH-III phase. A rough estimate thereof can be done in the following manner: at comparatively low pressures $(P \leq 5 \mathrm{GPa})$ we can consider the methane molecule as an approximate free rotor which yields the quantity $T S \approx 97 \mathrm{meV}$, while at intermediate pressures $(5<P<20 \mathrm{GPa})$ the molecule will explore the 4 possible orientations and $T S \approx 35 \mathrm{meV}$. At higher pressures, when rotational locking-in is effective ${ }^{10}$, only one orientation per molecule is possible and the corresponding entropy vanishes. This is used to estimate $\Delta G$ in figure 2 .

To summarize this comment, two main terms trigger the MH-III to MH-IV transition: the pressure term $P \Delta V$ and the ice cage internal energy.

It is therefore of interest to look into the ice network in greater detail. The main difference between MH-III and MH-IV is the presence of 4 -fold ans 8-fold rings in the former phase. In the following, we analyze the stability of the ice frames in MH-III and MH-IV in terms of the ice rings they consist of.gmail In order to carry out such an analysis, we cut out some non-equivalent rings from the ice frames that have been optimized at different pressures for both MH-III and MH-IV phases. Then, we replace the rings in a large supercell (about $20 \AA$ wide, so that the smallest distance between atoms within distinct periodic images is around $15 \AA$ ) and optimize their structure with the oxygen positions frozen at their initial values in the crystal. Therefore, the O-O distances are reminiscent of the pressure in the original crystal, while the deuterons are free to rearrange.

In Table II (top), we report the total energies of the 


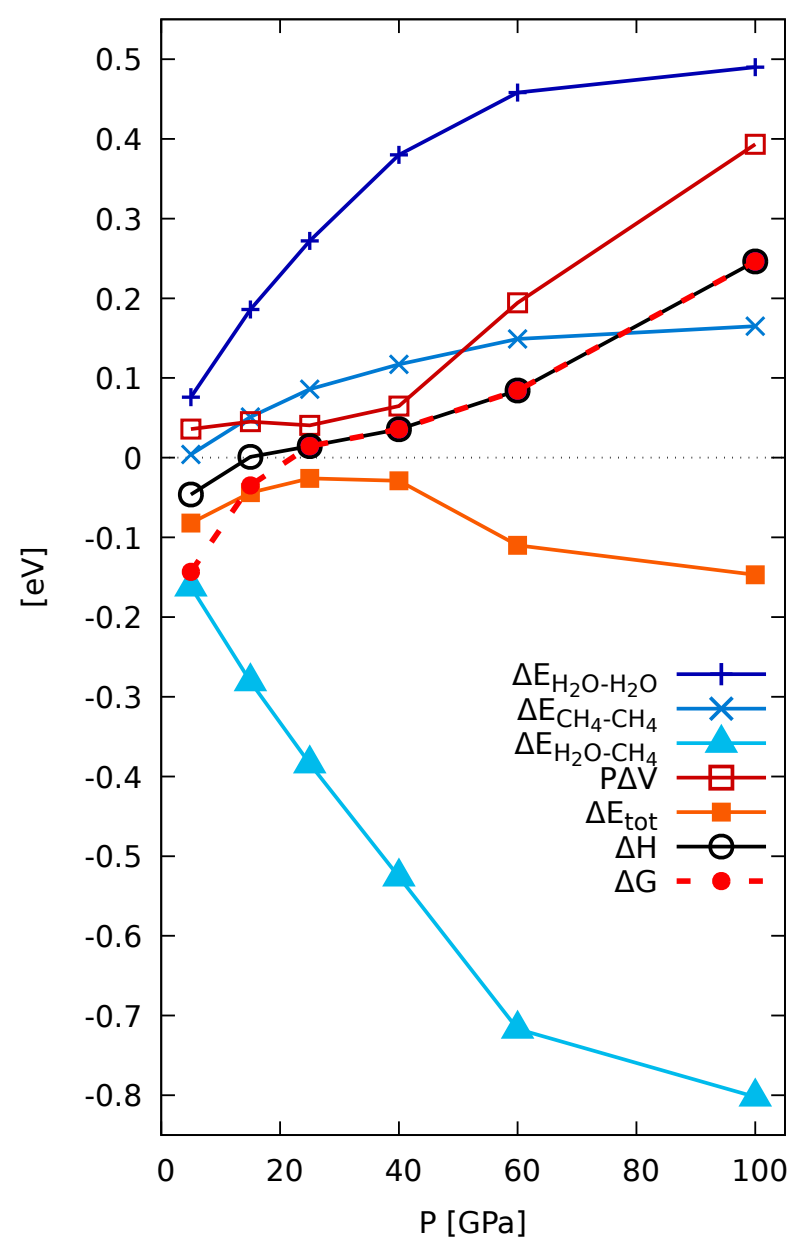

FIG. 2. Difference of the ice-ice, methane-methane, icemethane and $P V$ energies between MH-III and MH-IV, as defined in the text. A negative value implies that the contribution is larger in MH-III than in MH-IV. Values are in $\mathrm{eV}$ per unit formula $\mathrm{CH}_{4}:\left(\mathrm{D}_{2} \mathrm{O}\right)_{2}$.

rings that have been obtained in that way ${ }^{17}$. The fourth column on the right reports the energy of the rings where all atoms (both $\mathrm{O}$ and $\mathrm{H}$ ) are free to relax. By starting from the structural motifs of the $\mathrm{MH}$ crystals, we found a square for $\left(\mathrm{H}_{2} \mathrm{O}\right)_{4}$, the "book" and "cyclic" isomers for $\left(\mathrm{H}_{2} \mathrm{O}\right)_{6}$ and the "accordion" isomer for $\left(\mathrm{H}_{2} \mathrm{O}\right)_{8}$ (Fig.3). Those isomers have been also found in previous studies of water clusters ${ }^{18}$; we stress that here we are not interested in finding the lowest-energy $\left(\mathrm{H}_{2} \mathrm{O}\right)_{n}$ isomer for $n=4,6,8$, but to obtain the local minimum when starting from the constrained rings that were cut from the methane hydrate structures, within the very same computational scheme.

In the lower part of table II, we list the O-O distances within the various clusters. One can see that the $\mathrm{O}-\mathrm{O}$ distances are significantly shorter in comparison to the isolated, fully optimized, rings. For instance, the shortest $\mathrm{O}-\mathrm{O}$ distance in 4 -fold rings, decreases from $2.65 \AA$ in the isolated cluster, down to $2.46 \AA$ (MH-III, $20 \mathrm{GPa}$ ) and $2.28 \AA$ (MH-III, $60 \mathrm{GPa}$ ), a very small value. We also note that the $\mathrm{O}-\mathrm{O}$ distances in the topologically equivalent 6-fold rings (armchair rings in MH-III and MH-IV) are systematically longer in MH-IV than in MH-III. This
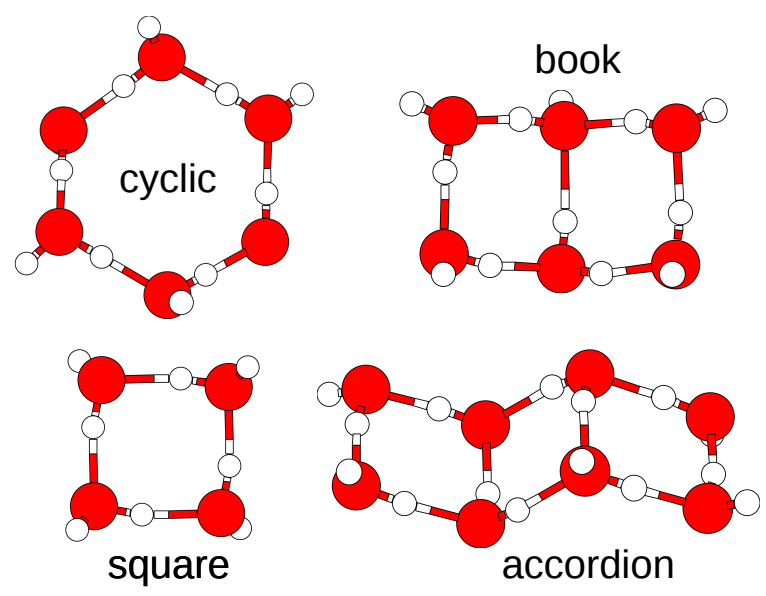

FIG. 3. Fully relaxed $\left(\mathrm{H}_{2} \mathrm{O}\right)_{n}$ clusters $(n=4,6,8)$ as obtained in our simulations. $\left(\mathrm{H}_{2} \mathrm{O}\right)_{4}$ square, $\left(\mathrm{H}_{2} \mathrm{O}\right)_{6}$ cycle, $\left(\mathrm{H}_{2} \mathrm{O}\right)_{6}$ book and $\left(\mathrm{H}_{2} \mathrm{O}\right)_{8}$ accordion. $\mathrm{O}$ are red and $\mathrm{H}$ white. The $\left(\mathrm{H}_{2} \mathrm{O}\right)_{6}$ cyclic isomer was obtained by geometry optimization starting from the "boat" 6b-member ring cut from the $\mathrm{MH}$ IV crystal, $\left(\mathrm{H}_{2} \mathrm{O}\right)_{6}$ book from the "armchair" 6a-member ring and $\left(\mathrm{H}_{2} \mathrm{O}\right)_{8}$ accordion from the 8-member ring in MH-III.

TABLE II. Cohesive energy and O-O distances of different kinds of ice clusters: 4-member, 6-member $(\mathrm{a}=$ armchair or $\mathrm{b}=$ boat) and 8-member rings, in $\mathrm{eV}$ per $\mathrm{H}_{2} \mathrm{O}$ molecule. The zero of the energy is that of an isolated water molecule. Second and third columns report the cohesive energy of ice rings obtained from $\mathrm{MH}-\mathrm{III}$ and $\mathrm{MH}-\mathrm{IV}$, where $\mathrm{O}$ atoms are clamped at their values in the respective crystals (MH-III or MH-IV) at the indicated pressures (20 or $60 \mathrm{GPa}$ ). In the fourth column, we report the cohesive energy of fully optimized rings. 6a rings relaxed to the "book" $\left(\mathrm{H}_{2} \mathrm{O}\right)_{6}$ isomer, $6 \mathrm{~b}$ rings to the $\left(\mathrm{H}_{2} \mathrm{O}\right)_{6}$ cyclic isomer and 8 rings to the "accordion" $\left(\mathrm{H}_{2} \mathrm{O}\right)_{8}$ isomer, which are sketched in Fig.3.

\begin{tabular}{llll}
\hline \hline & \multicolumn{3}{c}{ Cohesive energy $\left(\mathrm{eV} / \mathrm{H}_{2} \mathrm{O}\right)$} \\
cluster type & $P=60 \mathrm{GPa}$ & $P=20 \mathrm{GPa}$ & fully relaxed \\
\hline 4 (MH-III) & 0.177 & 0.348 & 0.386 \\
6a (MH-III) & 0.181 & 0.363 & 0.415 \\
8 (MH-III) & 0.201 & 0.367 & 0.442 \\
6a (MH-IV) & 0.270 & 0.391 & 0.415 \\
6b (MH-IV) & 0.262 & 0.380 & 0.407 \\
\hline \hline
\end{tabular}

\begin{tabular}{llll}
\hline \hline & \multicolumn{3}{c}{ O-O distances $(\AA)$} \\
cluster type & $P=60 \mathrm{GPa}$ & $P=20 \mathrm{GPa}$ & fully relaxed \\
\hline 4 (MH-III) & $2.32,2.28$ & $2.49,2.46$ & 2.65 \\
6a (MH-III) & $2.29,2.28$ & $2.46,2.44$ & \\
8 (MH-III) & $2.32,2.29,2.28$ & $2.49,2.46,2.44$ & $2.79,2.62$ \\
6a (MH-IV) & $2.37,2.35,2.33$ & $2.56,2.54,2.51$ & $2.84,2.60$ \\
6b (MH-IV) & $2.37,2.35,2.33$ & $2.56,2.54,2.51$ & 2.62 \\
\hline \hline
\end{tabular}

denotes a bigger strain on the rings composing $\mathrm{MH}-\mathrm{III}$ in comparison with MH-IV. These constraints are imposed by the external pressure and mainly differ because of the distinct ordering of the enclosed methane molecules in the two phases. In addition, the MH-IV rings are more symmetric and the O-O distances show less dispersion: they are thus closer to hexagonal symmetry and compatible with the high pressure structure in which the methane molecules are stacked along an effective 3-fold symme- 
try axis (Fig. 1). We also point out that in MH-III the O-O distances at $20 \mathrm{GPa}$ are just above the threshold value $2.42 \AA$ for the hydrogen-bond symmetrization, as established by Benoit and Marx ${ }^{19}$. Indeed, we observe deuteron centering between two $\mathrm{O}$ atoms in MH-III around $30 \mathrm{GPa}^{20}$.

Several trends are worth noting when comparing the energy of the same rings as a function of pressure in the corresponding crystals (Table II, top). The ring cohesive energy in MH-IV and MH-III decreases steadily with the constraints that are imposed by the external pressure and the distinct ordering of the enclosed methane molecules in the two phases. Indeed, as the O-O distances are shortened under increasing pressure, the $\mathrm{O}-\mathrm{H}$ covalent bond weakens and the O...H hydrogen bond strengthens. Globally, the first trend prevails and the total energy of the $\mathrm{O}-\mathrm{H} . . \mathrm{O}$ configuration increases when moving away from the optimal O-O distances in the fully optimized clusters. The ring cohesive energy decreases faster in MH-III than in MH-IV, even for the topological equivalent 6member armchair rings, consistently with the smaller O$\mathrm{O}$ distances (and thus a greater stress) in MH-III than in $\mathrm{MH}-\mathrm{IV}$, at the same pressure. Moreover, the reduction of the cohesive energy with increasing pressure is especially severe for 4-member rings, which are found in MH-III only. This is due to a significant angular frustration in 4fold ice rings, where hydrogen bonds significantly deviate from linearity.

To summarize this section, the MH-IV ice skeleton is less affected by pressure than its MH-III counterpart. This is at the root of the larger high-pressure cohesive energy of the ice skeleton in MH-IV as compared to MHIII. Together with the smaller volume of MH-IV, this drives the methane hydrate towards the thermodynamically more stable MH-IV at high pressure.

\section{FROM MH-IV TO MH-III.}

In order to complete the picture of the structural phase transition, we investigated the transition path through different sampling schemes of the free energy landscape.

In the following, we mainly rely on the results of metadynamics (MTD) simulations ${ }^{21}$, performed with the Plumed plugin $^{22}$. As the two structures differ both by the kind of medium-range order of the ice network and the methane orientations, we adopted as collective variables representing the system configuration the path coordinates based on permutation-invariant vectors (PIV) ${ }^{15,23}$, the latter being constructed from the relative atomic positions and being invariant under permutations between equivalent atoms. The transformation is represented in a two-dimensional space $(s, z)$, where $s$ quantifies the progress of the transformation and $z$, in the orthogonal space, allows to represent distinct pathways ${ }^{24}$, where the coordinates are defined solely on the basis of the reference phases MH-III and MH-IV without intermediate structures. This method has been recently applied to study the phase diagram of water ${ }^{15}$; here we report a novel application to study transformations at very high pressure, where atomic force and total energy are derived from first principles within the DFT. We notice that the transformation induces small changes in the lattice parameters and volume (see Table I). We run the MTD simulations at constant volume (we adopted the MH-IV equilibrium lattice parameters that we compute by static $T=0 \mathrm{~K}$ structural optimization at $P=40 \mathrm{GPa}$ ) and constant temperature $(T=300 \mathrm{~K})$.

An initial path was obtained from $T=0 \mathrm{~K}$ calculations employing the Nudged Elastic Band $(\mathrm{NEB})^{25}$; the path was then refined through metadynamics simulations. The transition is triggered by the weakening of the hydrogen bonds as $\mathrm{O}-\mathrm{O}$ distances contract under the increasing pressure. Below a typical $2.42 \AA$ O-O distance, there is a deuteron centering between the two $\mathrm{O}$ atoms, which is largely determined by the quantum nature of the proton or deuteron itself ${ }^{19}$; We thus took into account nuclear quantum effects (NQE) in a perturbative way, that is, along the path given by the classical simulations, by using path-integral simulation in the PIGLET implementation ${ }^{16}$. Specifically, we fixed the $\mathrm{O}$ atomic positions at those of selected configurations along the classical path, letting all other atoms free to move and performed PIMD, in order to estimate the corrections to the total energy connected to the quantum nature of the nuclei, in particular protons and deuterons. Although not quantitative, this procedure highlights the role of NQE in decreasing the energy barrier, mainly through the deuteron delocalization.

As shown in figure 4, the reaction path from MH-IV to MH-III provided by metadynamics is complex and passes through several stages:

(1) Between $s=1$ and $s=1.6$ a rather long and deep free energy well is found and correspond to structures within the MH-IV basin, which differ by hydrogen bond disorder.

(2) For $1.6 \leq s \leq 1.7$, the transition path shows a peculiar trend and varies mostly along $z$, almost normally to the linear NEB path connecting MH-IV to MH-III. This stage is characterized by the change of the orientation of roughly a half of the methane molecules. Methane ordering in MH-IV follows an $\mathrm{A} / \mathrm{B} / \mathrm{A}$... stacking along $\vec{b}$, where A describes methane molecule oriented upward along $\vec{z}$ and $\mathrm{B}$ downward along the same direction. Half of the methane molecules in this $(s, z)$ region rotate, inducing an $\mathrm{A} / \mathrm{A} / \mathrm{A}$ ordering along $\vec{b}$. This is the onset of the typical MH-III methane ordering. This happens also along the NEB reaction coordinate but, according to metadynamics, the precise rotations of methane molecules are different and occur before any hydrogen bond breaking or formation, in contrast with the NEB sequence.

(3) At $s \simeq 1.7, z \simeq 0.4$, metadynamics passes through a narrow path. This is the transition state, which is related to a "seesaw" mechanism as far as the $\mathrm{O}$ atoms in the ice network are concerned. Following the methane rotation, some hydrogen bonds break down and other form, so that the ice network of MH-IV, which is formed by 6 -fold rings, reorganizes. In Fig. 4, we see the onset of 4fold water rings induced by the shortening of the related oxygen atoms with the consequent formation of hydrogen bonds, while other $\mathrm{O}$ atoms move apart from each other, 


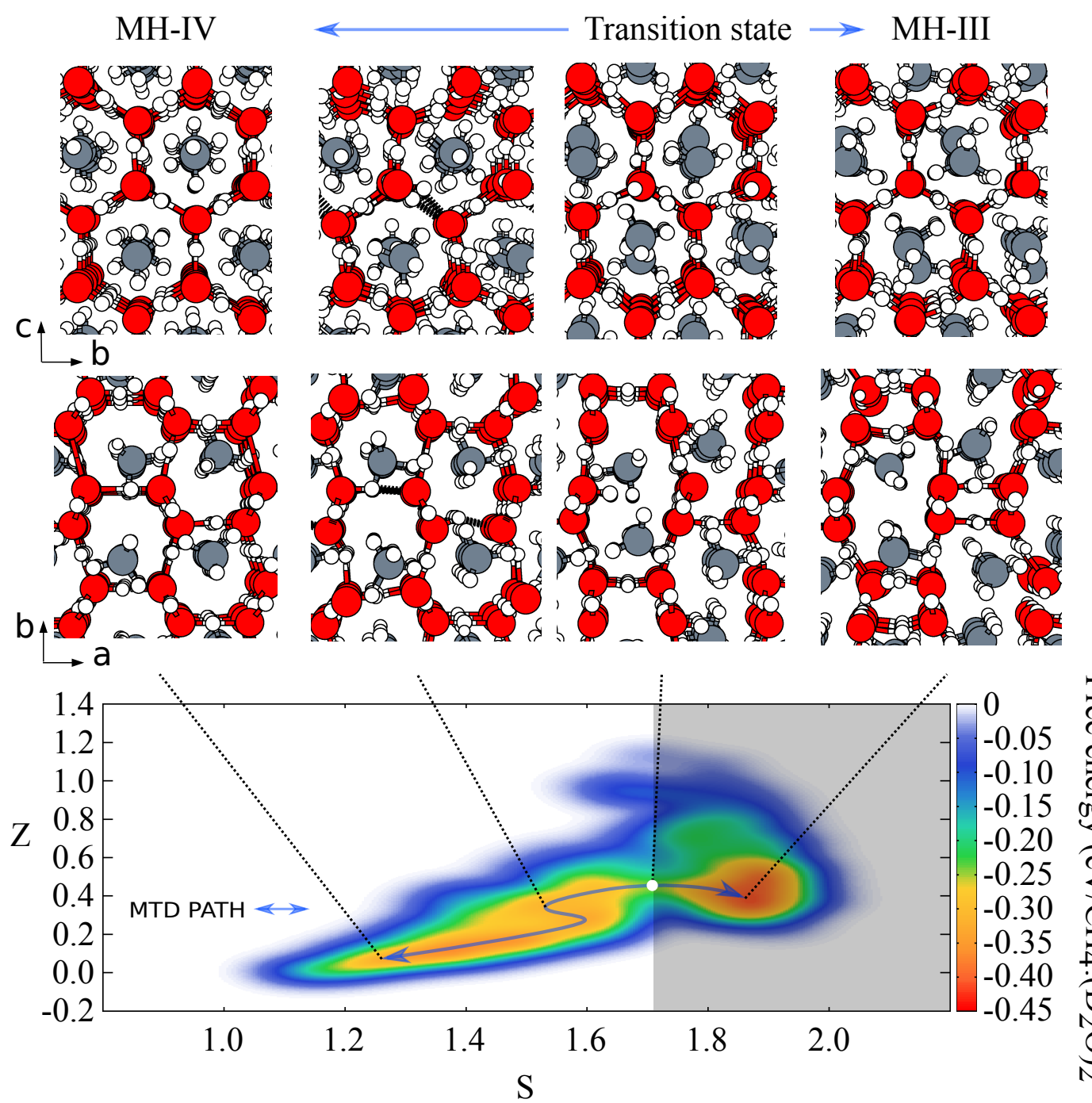

FIG. 4. Free energy landscape of the $(s, z)$ space as given by metadynamics, for the transition MH-IV $\rightarrow$ MH-III. The transition state is indicated with a white circle. The corresponding free energy barrier is estimated as $\Delta F^{*}=0.36 \mathrm{eV}$ per unit $\mathrm{CH}_{4}:\left(\mathrm{D}_{2} \mathrm{O}\right)_{2}$ formula. $s \simeq 1.3, z \simeq 0.1: \mathrm{MH}-\mathrm{IV} ; s \simeq 1.9, z \simeq 0.4:$ MH-III. The right part of the plot, shadowed in gray, corresponds to metadynamics bias in absence of a backward transition, hence it is not indicative of the backward free energy barrier MH$\mathrm{III} \rightarrow \mathrm{MH}-\mathrm{IV}$.

breaking the related hydrogen bonds and beginning the formation of 8-fold rings, in a seesaw fashion.

(4) Finally, for $1.8 \leq s \leq 2.0$, we recover the MHIII phase by completing the hydrogen bond reorganisation and the final reorientation of the methane molecules. The energy cost that is connected to those steps is small compared to the barrier, as the system moves within the MH-III basin.

According to our metadynamics simulations, the seesaw mechanism, which involves the displacement of the $\mathrm{O}$ atoms as well as some hydrogen bond breaking and formation, is the key point and the longest part of the transition, when adopting a classical picture for the nuclei. The corresponding free energy barrier amounts to $\Delta F^{*}=$ $0.36 \mathrm{eV} /$ unit formula, as estimated from the metadynamics bias deposited until the first passage through the transition state in absence of recrossing (hence a possible overestimation). All along the MTD runs, the diagonal components of the mean stress tensor are $\left\langle\sigma_{i i}\right\rangle=(46 \pm 1)$
GPa for MH-III and $\left\langle\sigma_{i i}\right\rangle=(41.5 \pm 0.5)$ GPa for MH$\mathrm{IV}$, for $i=1,2,3$ (off-diagonal terms are negligible). Therefore, the internal stress of the system is essentially isotropic and close to the nominal $40 \mathrm{GPa}$ pressure, although slightly different in the regions corresponding to the two distinct phases. According to the equation of state that we computed from the static $T=0 \mathrm{~K}$ structural optimizations, our approximation provides a fairly good estimate of the corresponding free energy barrier at constant pressure, within $\simeq 0.05 \mathrm{eV} /$ unit formula.

Whereas the displacement of the $\mathrm{O}$ atoms is little affected by NQE, the quantum deuteron delocalization plays a key role in the hydrogen bond breaking and formation. In the MH-III phase at $P=40 \mathrm{GPa}$, the $\mathrm{O}-\mathrm{O}$ distance is below the $2.42 \AA$ threshold that is commonly expected for the $\mathrm{H}$-bond centering ${ }^{19}$ and slightly above in the MH-IV phase, thus in the regime of deep deuteron tunneling. Therefore, when introducing nuclear quantum effects (NQE) through PIMD along the reaction trajec- 
tory, although in an approximate way (at fixed O positions), we observe a decrease of the energy barrier as compared with the $T=0 \mathrm{~K} \mathrm{NEB}$ calculation at the transition state of about $0.15 \mathrm{eV} /$ unit formula. Thus, we expect the reaction barrier found with MTD simulations to be downshifted by roughly the same value at $\Delta F^{*} \simeq 0.2$ $\mathrm{eV} /$ unit formula. Although this estimate is not definitive, as a precise evaluation of the free energy barrier including NQE was not possible for computational reasons, we guess that the MH-IV to MH-III transition might be allowed at $40 \mathrm{GPa}$, though possibly activated, as well as the reverse one, which was experimentally observed ${ }^{8}$.

\section{CONCLUSIONS}

Methane hydrate shows a remarkable stability at extreme pressures, exceeding $100 \mathrm{GPa}$, as indicated by recent Raman spectroscopy and first-principles simulations. Around $40 \mathrm{GPa}$, methane hydrate undergoes a transition from phase III to a new phase that we recently discovered, named IV (high-pressure). In the MHIV phase, in contrast with all the clathrates and hydrates that have previously been observed, the ice network meets that of pure ice at ordinary conditions.

These observations, which displace the presently known stability limit of hydrates, call for a detailed analysis of the role of the various interactions in the hydrate, namely the attractive water-water interaction, the repulsive water-methane and methane-methane interactions and the role of the external pressure, which drives the transition from the less compact MH-III phase to the denser MH-IV structure. The IV structure is formed by six-fold water rings enclosing the strongly constrained methane molecules. This arrangement optimizes the bonds and the angles between the water molecules in a compact arrangement, which maximizes the attractive water-water interaction. The channels that are left between the hexagonal ice rings are filled by ordered methane molecules, which raises the repulsive interaction with the ice network but at the same time generates a compact structure preventing it to collapse or dissociate. By increasing the pressure, the tight ice-methane arrangement within the IV phase prevails over the MHIII phase. The tetrahedral conformation of methane is a key factor for such optimal space filling; whenever the ice network was filled with spherical atoms having comparable size with the $\mathrm{CH}_{4}$ molecules, such as $\mathrm{Kr}$, the whole IV structure collapsed. Therefore, the combination of $\mathrm{H}_{2} \mathrm{O}$ molecules, tetrahedrally bound in six-fold rings, with tetrahedral $\mathrm{CH}_{4}$ molecules filling the ice channels constitutes a compact arrangement that is able to resist extreme pressures.

We also explored the complex transformation path from MH-IV to MH-III around $40 \mathrm{GPa}$, which is the experimental pressure for the MH-III to MH-IV transition. Along the transition, some hydrogen bonds break, allowing the six-fold rings in MH-IV to reorganize into 4-fold and 8-fold rings in MH-III. The 4-fold water rings are only stable at lower pressures than $40 \mathrm{GPa}$, as they are composed of strongly distorted hydrogen bonds. At the same time, the methane molecules change their orientation and their centres are slightly displaced. This complex transition is far from following the virtual linear path joining the MH-IV and MH-III motifs and only the exploration of the allowed configuration space via metadynamics provided a viable representation of the transition itself, with an approximate free energy barrier of $0.36 \mathrm{eV}$. Nuclear quantum effects are very significant all along the transition: the deuteron delocalization triggers the transition, allowing the hydrogen bonds to break and reform more gently than in the classical framework; at the same time, the transition barrier is lowered down to $\Delta F^{*} \simeq 0.2 \mathrm{eV} /$ unit formula.

We believe that our results, which show the interplay of the different interactions (attractive, repulsive) as well as the quantum delocalization of the light nuclei like $\mathrm{H}$ and $\mathrm{D}$, on the relative stability of the two phases and the transition barrier could be relevant not only for hydrates, but also for other water-based compounds at high pressures.

\section{ACKNOWLEDGEMENTS}

This work was granted access to the IVC resources of CINES under the allocation A0050906719 made by GENCI. We thank M. Benoit, C. Bonhomme, L. Bove and J. Teixeira for fruitful discussions.

${ }^{1}$ J. E. Dendy Sloan and C. A. Koh, Clathrate Hydrates of Natural Gases (CRC Press, 2007).

${ }^{2}$ M. Mitarai, M. Kishimoto, D. Suh, and R. Ohmura, Cryst. Growth Des. 15, 812 (2015).

${ }^{3}$ P. M. Naullage, A. A. Bertolazzo, and V. Molinero, ACS Cent. Sci. 5, 428 (2019).

${ }^{4}$ A. Geiger, A. Rahman, and F. H. Stillinger, J. Chem. Phys. 70, 263 (1979).

${ }^{5}$ R. Ludwig, Angew. Chem. Int. Ed. 40, 1808 (2001).

${ }^{6}$ C. A. Koh, Chem. Soc. Rev. 31, 157 (2002).

${ }^{7}$ J. S. Loveday and R. J. Nelmes, Phys. Chem. Chem. Phys. 10, 937 (2008).

${ }^{8}$ S. Schaack, U. Ranieri, P. Depondt, R. Gaal, W. K. Kuhs, P. Gillet, F. Finocchi, and L. E. Bove, Proc. Natl. Acad. Sci. U.S.A. 116 (33), 16204 (2019).

${ }^{9}$ J. Loveday, R. J. Nelmes, M. Guthrie, D. D. Klug, and J. S. Tse, Phys. Rev. Lett. 87, 215501 (2001).

${ }^{10}$ S. Schaack, U. Ranieri, P. Depondt, R. Gaal, W. F. Kuhs, A. Falenty, P. Gillet, F. Finocchi, and L. E. Bove, J. Phys. Chem. C 122, 11159 (2018).

${ }^{11}$ H. Hirai, S.-I. Machida, T. Kawamura, Y. Yamamoto, and T. Yagi, Amer. Mineral. 91, 826 (2006).

${ }^{12}$ P. Giannozzi, S. Baroni, N. Bonini, M. Calandra, R. Car, C. Cavazzoni, D. Ceresoli, G. L. Chiarotti, M. Cococcioni, I. Dabo, A. Dal Corso, S. de Gironcoli, S. Fabris, G. Fratesi, R. Gebauer, U. Gerstmann, C. Gougoussis, A. Kokalj, M. Lazzeri, L. Martin-Samos, N. Marzari, F. Mauri, R. Mazzarello, S. Paolini, A. Pasquarello, L. Paulatto, C. Sbraccia, S. Scandolo, G. Sclauzero, A. P. Seitsonen, A. Smogunov, P. Umari, and R. M. Wentzcovitch, J. Phys.: Cond. Matt. 21, 395502 (2009).

${ }^{13}$ J. P. Perdew, K. Burke, and M. Ernzerhof, Phys. Rev. Lett. 77, 3865 (1996).

${ }^{14}$ S. Grimme, J. Comput. Chem. 27, 1787 (2006).

${ }^{15}$ S. Pipolo, M. Salanne, G. Ferlat, S. Klotz, A. M. Saitta, and F. Pietrucci, Phys. Rev. Lett. 119, 245701 (2017).

${ }^{16} \mathrm{M}$. Ceriotti and D. E. Manolopoulos, Phys. Rev. Lett. 109, 100604 (2012). 
${ }^{17}$ For instance, a 6a-member (armchair) ring in MH-IV at $20 \mathrm{GPa}$ was cut from the MH-IV crystal at $20 \mathrm{GPa}$, then optimized in the big supercell at frozen oxygen positions.

${ }^{18}$ H. M. Lee, S. B. Suh, J. Y. Lee, P. Tarakeshwar, and K. S. Kim, J. Chem. Phys. 112, 9759 (2000).

${ }^{19}$ M. Benoit and D. Marx, ChemPhysChem 6, 1738 (2005).

${ }^{20}$ S. Schaack, P. Depondt, and F. Finocchi, J. Phys.: Conf. Series 1136, 012018 (2018).

${ }^{21}$ A. Laio and M. Parrinello, Proc. Natl. Acad. Sci. U.S.A. 99, 12562 (2002).
${ }^{22}$ G. A. Tribello, M. Bonomi, D. Branduardi, C. Camilloni, and G. Bussi, Comp. Phys. Comm. 185, 604 (2014).

${ }^{23}$ G. A. Gallet and F. Pietrucci, J. Chem. Phys. 139, 074101 (2013).

${ }^{24}$ D. Branduardi, F. L. Gervasio, and M. Parrinello, J. Chem. Phys. 126, 054103 (2007).

${ }^{25}$ H. Jónsson, G. Mills, and K. W. Jacobsen, "Nudged elastic band method for finding minimum energy paths of transitions," in Classical and Quantum Dynamics in Condensed Phase Simulations, edited by G. C. B. J. Berne and D. F. Coker (World Scientific, Singapore, 1998). 